\title{
Localized-Density-Matrix Method and Its Application to Carbon Nanotubes
}

\author{
WanZhen Liang, Satoshi Yokojima, DongHao Zhou, and GuanHua Chen* \\ Department of Chemistry, The University of Hong Kong, Pokfulam Road, Hong Kong \\ Received: March 8, 1999; In Final Form: June 2, 1999
}

\begin{abstract}
The localized-density-matrix method (Yokojima, S.; Chen, G. H. Chem. Phys. Lett. 1998, 292, 379) is employed to simulate the optical responses of very large carbon nanotubes and polyacetylene oligomers containing 10000 carbon atoms. The Pariser-Parr-Pople Hamiltonian is used to describe the $\pi$ electrons in these systems, and the time-dependent Hartree-Fock approximation is employed to calculate the linear optical responses. In the calculation, the fast multipole method or the cell multipole method is employed to evaluate the effects of Coulomb interaction. It is illustrated that the computational time scales linearly with the system size for carbon nanotubes while high accuracy is achieved.
\end{abstract}

\section{Introduction}

Recently the localized-density-matrix (LDM) method has been developed to evaluate ground- and excited-state properties. $^{1-5}$ It is based on the truncation of the reduced single-electron density matrices. The computational time of the LDM method scales linearly with the system size $N$. The method has been tested successfully for evaluating the optical properties of conjugated polymers. The time-dependent Hartree-Fock (TDHF) approximation $^{6}$ has been employed in the calculations. ${ }^{1-5}$ In refs $1-5$, a cutoff method is used to evaluate the summation of Coulomb interactions

$$
\sum_{k} v_{i k} \rho_{k k}
$$

where $v_{i k}$ is the Coulomb interaction between two electrons at local orbitals $i$ and $k$ separately and $\rho$ is the reduced density matrix. The cutoff was introduced to achieve the overall $O(N)$ scaling of the LDM method, 1,2 since the straightforward evaluation of eq 1 for all possible $i$ scales as $O\left(N^{2}\right)$. Accurate results have been obtained as compared with those of the full TDHF calculation, and this is due to the cancellation of errors for structurely ordered molecules such as polyacetylene. ${ }^{1,2}$ However, many physical, chemical, and biological systems do not possess such ordered structures. The cutoff approximation may thus lead to numerical error. ${ }^{7,8}$ A variety of approximate methods have been used to calculate accurately the Coulomb interaction such as eq 1.9,10 The fast multipole method (FMM) or cell multipole method (CMM) divides the system into boxes of different sizes, and replaces the pairwise Coulomb interaction with multipolar expansions. This leads the $O(N)$ scaling of the computational time, and thus reduces drastically the computational time for large systems. ${ }^{7,8,11-14}$

Conjugated $\pi$ electronic systems possess many interesting electric and optical properties. These properties arise mainly from the dynamics of $\pi$ electrons in these systems. Conjugated polymers have large nonlinear optical responses and may be used for the optical devices. Many experimental ${ }^{15-17}$ and theoretical ${ }^{18-20}$ works have been devoted to investigate their electronic structures. Polyacetylene has been used as a model

* To whom correspondence should be addressed. system to understand the electronic and nuclear dynamics of the $\pi$ conjugated polymeric systems. It is often employed to calibrate the validity and accuracy of new theoretical methods. Carbon nanotubes (CNTs) are graphite sheets rolled up along some axis. ${ }^{21}$ They are a new class of materials with a reduced dimensionality and are expected to possess very interesting mechanical properties, such as high stiffness and axial strength, and magnetic, optical, and transport properties. ${ }^{22-32}$ They are made of coaxial graphite cylinders with nanometer scale radii and micrometer scale lengths, and are thus ideal quasi-onedimensional systems for investigating the fundamental issues of one-dimensional systems. Various experiments carried out thus far (transmission electron spectroscopy (TEM), ${ }^{21,23,33,34}$ scanning tunneling microscopy and spectroscopy (STM/ STS), ${ }^{24-28}$ and Raman scattering ${ }^{35-37}$ ) consistently show that the CNTs are cylindrical graphene sheets of $\mathrm{sp}^{2}$-bonded carbon atoms.

Despite the short time since the first CNTs were synthesized, possible applications of the new materials have been reported, such as atomic scale field emitters or pinning materials in hightemperature superconductors. ${ }^{38,39}$ They also have very interesting optical properties and large nonlinear optical responses and may be used for electro-optical devices. It is thus important to probe the electronic properties of these CNTs. It has been observed ${ }^{34}$ that the tubules can be formed from a single layer of graphite. Such tubules would be expected to have unique properties. Single-wall nanotubes are the best systems for investigating the intrinsic properties of this new material class. Theoretical predictions of the electronic structure of CNTs regarding their metallic and semiconducting properties depend strongly on the tubule diameter and chiralities. ${ }^{23,40}$ Some of those theoretical predictions have been confirmed experimentally, such as in STM/STS ${ }^{26-28}$ (which reveal the electronic structure and crystalline structure) and electron energy loss spectroscopy $(\mathrm{EELS})^{41,42}$ (which determines the electronic band structure). The optical properties of CNTs have been measured, and a broad absorption peak has been observed near $1.17 \mathrm{eV} .{ }^{43}$ Theoretical calculations of the optical spectra have been carried. ${ }^{44,45}$ However, these calculations were based on Hubbard-like models and were carried out at the Hartree-Fock level. Better Hamiltonians and more accurate methods which include electronelectron correlation are thus warranted. 
In this work we modify the current LDM method by incorporating the FMM or CMM into our existing computer code, and calculate the ground- and excited-state properties of very large polyacetylene oligomers and zigzag $(m, 0)$ CNTs. In section 2 we briefly describe the PPP models for polyacetylene oligomers and CNTs, and outline the TDHF method for linear optical response. In section 3 the LDM formalisms for the ground and excited states are given, and the FMM or CMM approximation for eq 1 is described. The absorption spectra of polyacetylene oligomers containing up to 10000 carbon atoms are examined in section 4. The calculations for CNTs are outlined in section 5 along with the corresponding results. Finally a discussion and conclusion are given in section 6 .

\section{Model and Time-Dependent Hartree-Fock Approximation}

We can consider only the $\pi$ electrons in the conjugated systems since the electric and optical properties come mainly from the responses of $\pi$ electrons to the external fields. The $\pi$ electrons of conjugated systems in the external field $\vec{E}$ are well described by the Pariser-Parr-Pople (PPP) Hamiltonian ${ }^{18-20}$

$$
\begin{gathered}
H=H_{\mathrm{e}}+H_{\mathrm{ee}}+H_{\mathrm{ext}} \\
H_{\mathrm{e}}=\sum_{m n} t_{m n} a_{m}^{\dagger} a_{n} \\
H_{\mathrm{ee}}=\sum_{m n} V_{m n} a_{m}^{\dagger} a_{m} a_{n}^{\dagger} a_{n} \\
H_{\mathrm{ext}}=-\mathrm{e} \vec{E} \cdot \sum_{m n} \vec{\mu}_{m n} a_{m}^{\dagger} a_{n}
\end{gathered}
$$

where $a_{m}^{\dagger}\left(a_{n}\right)$ is the creation (annihilation) operator for an electron at the localized atomic orbital $m(n) . H_{\text {ext }}$ is the interaction between the $\pi$ electrons and an external electric field $\vec{E}(t)$, and $\vec{\mu}$ is the dipole moment matrix. $H_{\mathrm{ee}}$ is the two-electron part of the Hamiltonian which represents the effective Coulomb interaction between two $\pi$ electrons. The zero differential overlap (ZDO) approximation ${ }^{46}$ is used here. $V_{m n}$ is the electron-electron Coulomb interaction between two electrons at local orbitals $m$ and $n$, respectively, and may be expressed by the Ohno formula ${ }^{47}$

$$
V_{m n}=\frac{U_{0} / \epsilon}{\left[1+\left(r_{m n} / a_{0}\right)^{2}\right]^{1 / 2}}
$$

where $r_{m n}$ is the distance between two orbitals $m$ and $n$ and $U_{0} / \epsilon$ is the on-site Coulomb repulsion. $\epsilon$ is the static dielectric constant caused by the polarization of core and $\sigma$ electrons. $a_{0}$ is of the same magnitude as the bond length. In the calculation $U_{0}=11.13 \mathrm{eV}, \epsilon=1.5$, and $a_{0}=1.2935 \AA$ are used. $H_{\mathrm{e}}$ is the one-electron part of the Hamiltonian which describes the dynamics of a single electron in the absence of other electrons. The diagonal elements $t_{m m}$ represent the energy of the electron in orbital $m$, whereas the off-diagonal element $t_{m n}$ is the hopping matrix element between $m$ and $n$. In the calculations $t_{m m}$ is set to zero while $t_{m n}(m \neq n)$ is of different form for polyacetylene and CNTs. For polyacetylene

$$
t_{m n}= \begin{cases}\bar{\beta}-\beta^{\prime} \Delta z_{n} & m=n \pm 1 \\ 0 & \text { otherwise }\end{cases}
$$

where $\Delta z_{n}$ is the bond length deviation from the equilibrium bond length which is $1.41 \AA$ A. $\bar{\beta}$ and $\beta^{\prime}$ are set to $-2.4 \mathrm{eV}$ and $-3.5 \mathrm{eV} \cdot \AA^{-1}$, respectively. ${ }^{19}$ The geometrical structure of a
CNT is uniquely determined by its chiral vector $\vec{R}=m \vec{a}_{1}+$ $n \vec{a}_{2}$ around the circumference of the nanotubes. $\vec{a}_{1}$ and $\vec{a}_{2}$ are the unit vectors of the graphene $2 \mathrm{D}$ lattice. The tubule diameter $D_{t}$ is simply related to the integer pair $(m, n)$ by $D_{t}=\sqrt{ } 3 a_{\mathrm{C}-\mathrm{C}}\left(m^{2}\right.$ $\left.+m n+n^{2}\right)^{1 / 2} / \pi$. The nearest-neighbor carbon-carbon distance $a_{\mathrm{C}-\mathrm{C}}$ is chosen to be $1.421 \AA$. The axis of the CNTs is chosen along the $z$ direction in our calculations. Zigzag $(m, 0)$ CNTs are the graphite sheets which are curved along the rolled-up direction. A different form is thus required.

$$
t_{i j}=\left\{\begin{array}{lc}
0 & \text { atoms } i \text { and } j \text { are not bonded } \\
-\gamma_{0}\left(1+1 / m^{2}\right) & \text { atoms } i \text { and } j \text { are bonded in the } \\
-\gamma_{0} & \text { rolled-up direction } \\
& \begin{array}{c}
\text { atoms } i \text { and } j \text { are bonded along the } \\
\text { tube axis direction }
\end{array}
\end{array}\right.
$$

Here $\gamma_{0}$ is set to $2.5 \mathrm{eV}$ which is the same as that for the graphite. For nanotubes with very small radii, the electron correlation effects cannot be neglected. Thus, the hopping matrix elements along and around the tubule differ slightly, by an amount on the order of $1 / \mathrm{m}^{2} .48$

Within the TDHF approximation, the reduced single-electron density matrix satisfies the following relation:

$$
i \hbar \frac{\mathrm{d}}{\mathrm{d} t} \rho(t)=[h(t)+f(t), \rho(t)]
$$

where $h(t)$ is the Fock matrix and $f(t)$ describes the interaction between an electron and the external field $\vec{E}(t),{ }^{19}$ which is parallel to the $z$ axis, and they may be expressed as

$$
\begin{gathered}
h_{n m}(t)=t_{n m}+2 \delta_{n m} \sum_{l} v_{n l} \rho_{l l}(t)-v_{n m} \rho_{n m}(t) \\
f_{n m}(t)=\delta_{n m} \mathrm{ez}(n) E(t)
\end{gathered}
$$

where $\delta_{m n}$ is a Kroenecker delta.

Considering the linear response only, $\rho$ may be expressed as

$$
\rho=\rho^{(0)}+\delta \rho^{(1)}
$$

where $\rho^{(0)}$ is the Hartree-Fock ground-state reduced density matrix for the PPP Hamiltonian $H$ when $E(t)=0$ (or the PPP Hartree-Fock ground-state density matrix) and $\rho^{(1)}$ is the firstorder induced density matrix in $E(t)$. Similarly, Fock matrix $h$ may be decomposed as

$$
h=h^{(0)}+\delta h^{(1)}
$$

where $h^{(0)}$ is the Fock matrix when $E(t)=0$ with

$$
h_{n m}^{(0)}(t)=t_{n m}+2 \delta_{n m} \sum_{l} v_{n l} \rho_{l l}^{(0)}(t)-v_{n m} \rho_{n m}^{(0)}(t)
$$

and $\delta h^{(1)}$ is the first-order induced Fock matrix when $E(t) \neq 0$

$$
\delta h_{n m}^{(1)}(t)=2 \delta_{n m} \sum_{l} v_{n l} \delta \rho_{l l}^{(1)}(t)-v_{n m} \delta \rho_{n m}^{(1)}(t)
$$

Equation 5 may thus be rewritten as

$$
\mathrm{i}\left(\hbar \frac{\mathrm{d}}{\mathrm{d} t}+\gamma\right) \delta \rho^{(1)}=\left[h^{(0)}, \delta \rho^{(1)}\right]+\left[\delta h^{(1)}, \rho^{(0)}\right]+\left[f, \rho^{(0)}\right]
$$


A phenomenological parameter $\gamma$ is introduced to simulate the dephasing process.

\section{Linear-Scaling LDM Method}

3.1. Ground State. The PPP Hartree-Fock ground-state density matrix $\rho^{(0)}$ may be determined by the equation

$$
\left[h^{(0)}, \rho^{(0)}\right]=0
$$

together with the idempotency condition

$$
\rho^{(0)} \rho^{(0)}=\rho^{(0)}
$$

The TDHF eq 5 is usually used to simulate the electronic response to the external field. It may also be used to determine $\rho^{(0)}$ by setting $\vec{E}(t)=0$ or $f(t)=0$. Starting from an initial guess of the density matrix $\rho(t)$ at time $t=t_{0}$, we follow its time evolution by integrating the equation

$$
\mathrm{i}\left(\hbar \frac{\mathrm{d}}{\mathrm{d} t}+\gamma\right) \rho(t)=[h(t), \rho(t)]
$$

$\gamma \equiv \gamma(t)$ is time-dependent, and approaches zero over time. It ensures the eventual convergence of eq 15 toward eq 13 or the convergence of $\rho(t)$ to $\rho^{(0)} \cdot \gamma(t)$ may be of different forms, and we choose

$$
\gamma(t)=\gamma_{0} \frac{1}{1+\left(t-t_{0}\right) / \bar{t}}
$$

where $\gamma_{0}$ is the dephasing parameter at $t=t_{0}$ and $\bar{t}$ is the time constant which is approximately the simulation time. Since $\rho^{(0)}$ is real, we retain only the real part of $\rho(t)$ as the current approximate density matrix after each integration of eq 15 over a time interval $\Delta t$. The idempotency condition is then imposed by applying repeatedly 49,50

$$
\rho^{\prime}=3(\rho)^{2}-2(\rho)^{3}
$$

until a convergence is reached. The resulting $\rho^{\prime}$ is then taken as the new approximate density matrix. The above process, which includes the integration of eq 15 over $\Delta t$, the retention of $\operatorname{Re}[\rho(t)]$, and imposing the idempotency, is applied iteratively until the density matrix $\rho(t)$ is converged. ${ }^{3,4}$ Note that the initial guess $\rho\left(t_{0}\right)$ is preferably close enough to the real ground-state density matrix $\rho^{(0)}$; otherwise a divergence may occur.

To achieve the linear-scaling computation for the ground state, we adopt the following approximation: ${ }^{3,4}$

$$
\rho_{i j}(t)=0 \text { for } r_{i j}>l_{1}
$$

where $r_{i j}$ is the distance between two atomic orbitals $i$ and $j$, and $l_{1}$ is a critical length for the density matrix. ${ }^{1,2}$ Consequently

$$
h_{i j}(t)=0 \text { for } r_{i j}>l_{1}
$$

In the calculation, the convergence criterion for eq 15 is given by

$$
\sum_{i j}\left|\sum_{k}\left(h_{i k} \rho_{k j}-\rho_{i k} h_{k j}\right)\right| / N<R_{1}
$$

and for eq 17

$$
\sum_{i j}\left|\rho_{i j}{ }^{\prime}-\rho_{i j}\right| / N<R_{2}
$$

$t_{0}=-0.05 \mathrm{fs}, \gamma_{0}=1.0 \times 10^{-6} \mathrm{eV}, \bar{t}=10 \mathrm{fs}, \Delta t=0.138 \mathrm{fs}$, $R_{1}=1.0 \times 10^{-3} \mathrm{eV}$, and $R_{2}=1.0 \times 10^{-8}$ have been employed.

3.2. Excited State. Equation 12 can be rewritten as

$$
\begin{aligned}
& \mathrm{i}\left(\hbar \frac{\mathrm{d}}{\mathrm{d} t}+\gamma\right) \delta \rho_{i j}^{(1)}=\sum_{k}\left(h_{i k}^{(0)} \delta \rho_{k j}^{(1)}-\delta \rho_{i k}^{(1)} h_{k j}^{(0)}\right)+ \\
& 2 \sum_{k}\left(v_{i k}-v_{j k}\right) \rho_{i j}^{(0)} \delta \rho_{k k}^{(1)}-\sum_{k}\left(v_{i k} \rho_{k j}^{(0)} \delta \rho_{i k}^{(1)}-v_{k j} \rho_{i k}^{(0)} \delta \rho_{k j}^{(1)}\right)+ \\
& \mathrm{e}(z(i)-z(j)) E(t) \rho_{i j}^{(0)}(20)
\end{aligned}
$$

Most matrix elements of $h^{(0)}, \rho^{(0)}$, and $\delta \rho^{(1)}$ are virtually zero when the distance between the two atoms is large enough. The following approximations are employed to achieve the linearscaling calculation for the excited-state properties:

$$
\begin{gathered}
\rho_{i j}^{(0)}=0 \text { if } r_{i j}>l_{0} \\
\delta \rho_{i j}^{(1)}=0 \text { if } r_{i j}>l_{1}
\end{gathered}
$$

Approximations A and B are the same as those in ref 1. An immediate consequence of (A) is that $h_{i j}^{(0)}=0$ when $r_{i j}>l_{0}$. With the truncation of the density matrix and Fock matrix, the computational time is finite for evaluating the first, third, and fourth terms on the right-hand side (rhs) of eq 20 for each $\delta \dot{\rho}_{i j}^{(1)}$. The FMM or CMM is employed to evaluate the second term, and the corresponding computational time is finite for each $\delta \dot{\rho}_{i j}^{(1)}$. Approximation B means that only $O(N)$ of $\delta \rho_{i j}^{(1)}$ needs to be considered explicitly. Thus, the overall computational time scales linearly with the system size.

In the calculation of the second term on the rhs of eq 20 , the quantity

$$
U(i)=\sum_{k} V_{i k} \delta \rho_{k k}^{(1)}=\sum_{k} q_{k} /\left(a_{0}^{2}+r_{i k}^{2}\right)^{1 / 2}
$$

is to be evaluated for each $i$, where $q_{k} \equiv a_{0} U_{0} \delta \rho_{k k}^{(1)} / \epsilon$. Straightforward computation of $U(i)$ requires $O\left(N^{2}\right)$ operations for all $i$. The FMM reduces the evaluation of all $U(i)$ to $O(N)$ operations. ${ }^{11,12,51}$ Details of the FMM procedure adopted in this work are given in the Appendix.

\section{Polyacetylene Oligomers}

To assess the accuracy of the FMM calculation, the Coulomb energy

$$
V=\frac{1}{2} \sum_{i \neq j} \frac{q_{i} q_{j}}{\left(a_{0}^{2}+r_{i j}^{2}\right)^{1 / 2}}
$$

has been calculated by both the FMM and the exact evaluation with $q_{i}=q_{j}=a_{0} U_{0} / \epsilon$. The relative errors are below $0.1 \%$ and saturate for $N>20000$. The computational accuracy is comparable to that of ref 8 , because $a_{0}$ is small. The saturation is due to the fact that the Coulomb energy decreases toward zero as the distance increases. We check the accuracy of eq 22 with $q_{i}=q_{j}=1$, set $a_{0}=0$, and find the error with $a_{0}=1.29$ $\AA$ is a little bit larger than that with $a_{0}=0$. The difference of the errors is about $0.003 \%$.

To evaluate the excited-state properties, the PPP HartreeFock ground-state density matrix $\rho^{(0)}$ must be calculated first. We plot the computational time for obtaining $\rho^{(0)}$ in Figure 1a. The computational time scales indeed linearly with the size $N$. The PPP Hartree-Fock ground-state energies are calculated by using both the cutoff method (denoted as the cutoff-LDM 

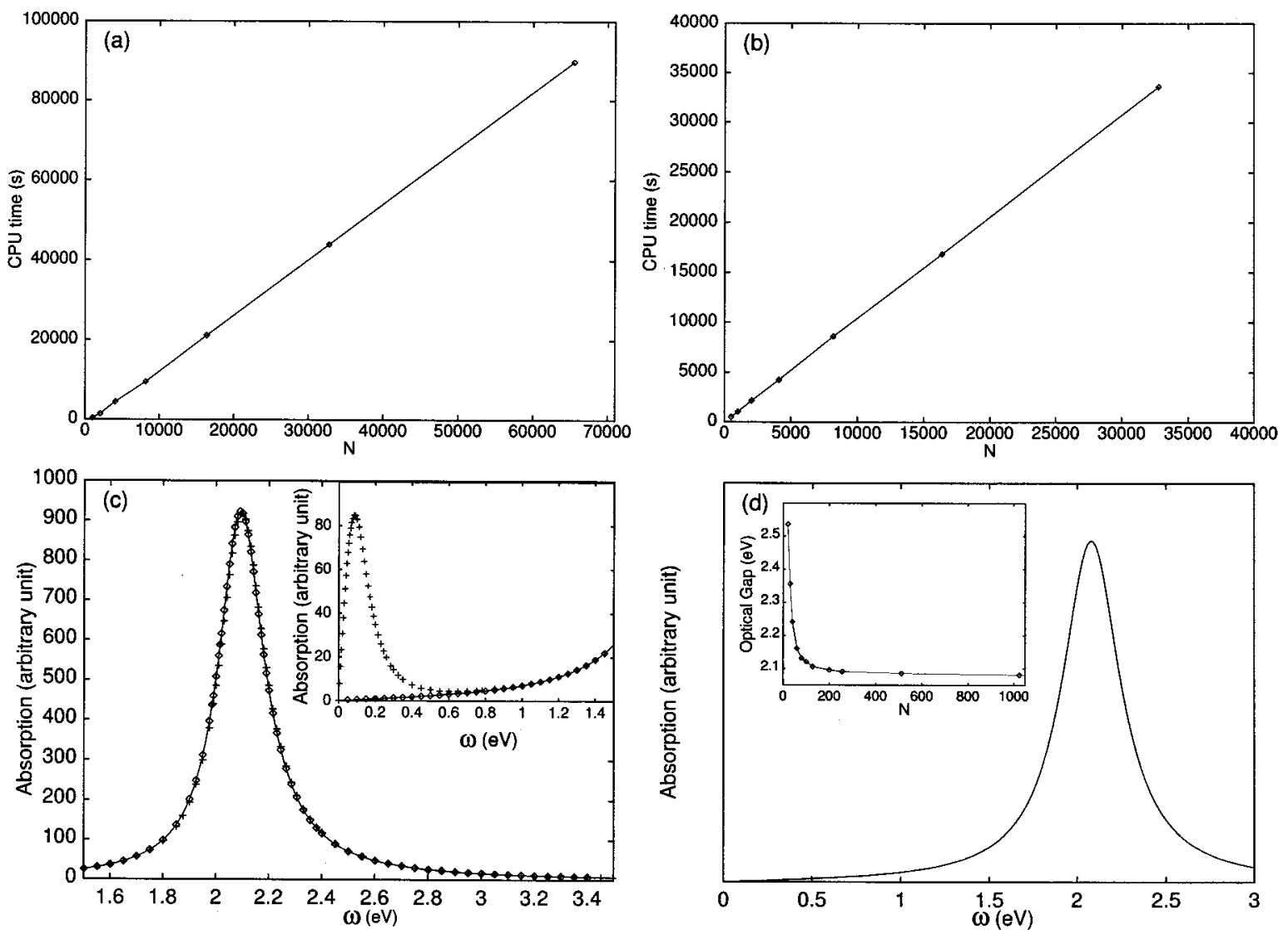

Figure 1. (a) CPU time versus $N$ for the calculation of ground-state reduced density matrices $\left(l_{1}=50 \AA\right.$ and 16 atoms in the smallest box). (b) CPU time for the calculation of the excited state $\left(l_{1}=l_{0}=37 \AA\right.$ and 16 atoms in the smallest box $)$. Each calculation is performed during a time interval of $[-0.5 \mathrm{fs},-0.3 \mathrm{fs}]$ with a time step of $0.01 \mathrm{fs}$. (c) Absorption spectrum for $N=200$. The crosses are the cutoff-LDM results with $l_{1}=$ $l_{0}=50 \AA$ and $l_{\mathrm{c}}=25 \AA$. The diamonds are the FMM-LDM results $l_{1}=l_{0}=50 \AA$ and 25 atoms in the smallest box. The solid lines are the full TDHF results. Each calculation is performed during the time interval between -0.5 and $\pm 70.0 \mathrm{fs}$ with a time step of $0.01 \mathrm{fs}$. $\gamma=0.1 \mathrm{eV}$. (d) Absorption spectra for $N=10000$ by using the FMM-LDM method with $l_{1}=l_{0}=50 \AA$. The 8 -level FMM is employed. The dephasing parameter $\gamma=0.2 \mathrm{eV}$.

hereafter) with $l_{\mathrm{c}}=37 \AA$ and the FMM (denoted as FMMLDM hereafter) with the smallest box containing 16 atoms for eq 1 . In both methods, $l_{1}$ is equal to $50 \AA$. The PPP HartreeFock ground-state energies calculated by the cutoff-LDM and the FMM-LDM methods are almost the same. This is because the nuclear charges and the net electron charges at each site cancel each other. The relative errors are $1.0 \times 10^{-7}$.

To calculate the absorption spectrum, an external field

$$
E(t)=(1 / \sqrt{ } \pi \bar{t}) \mathrm{e}^{-(t / \bar{t})^{2}}
$$

is employed, where $\bar{t}=0.1$ fs. The phenomenological dephasing constant $\gamma$ is set to $0.1 \mathrm{eV}$ in the calculation. The fourth-order Runge-Kutta method ${ }^{52}$ is used to integrate eq 20 . We compare the full TDHF, the FMM-LDM, and the cutoff-LDM methods for $N=200 . l_{0}=l_{1}=50 \AA$ is employed in both LDM calculations. A four-level hierarchy is used in the FMM calculation. The results are shown in Figure 1c. Clearly three sets of results are consistent for $\omega>0.8 \mathrm{eV}$. When $\omega<0.8$ $\mathrm{eV}$ as shown in the inset of Figure 1c, the FMM-LDM method yields much better results than the cutoff-LDM method with $l_{\mathrm{c}}$ $=25 \AA$. This is because the FMM-LDM method considers the Coulomb interaction in all the space of the system while the cutoff-LDM method neglects the influences of the charges outside the cutoff region. The distance of the charge movement is larger in the low-frequency region than that in the highfrequency region. The cutoff method ignores the charge fluctuations outside the cutoff region, and thus introduces extra errors.
The FMM-LDM method yields slightly more accurate values for the optical gap than the cutoff-LDM method. The difference of the two results is less than $0.006 \mathrm{eV}$. The relative error of the cutoff-LDM method for the optical gap is about $0.25 \%$. The FMM-LDM and the cutoff-LDM methods give much less relative numerical errors compared to the FMM or the cutoff calculations of eq 22. For instance, in the excited-state calculation, we need to evaluate the term $2 \sum_{k}\left(v_{i k}-v_{j k}\right) \rho_{i j}^{(0)} \delta \rho_{k k}^{(1)}$. The errors appearing in $\sum_{k} v_{i k} \rho_{i j}^{(0)} \delta \rho_{k k}^{(1)}$ and $\sum_{k} v_{j k} \rho_{i j}^{(0)} \delta \rho_{k k}^{(1)}$ cancel each other because of the system symmetry for both the FMM and the cutoff methods. Thus, we have relatively small errors in both methods.

In Figure 1b, we plot the CPU time of the FMM-LDM calculation versus $N$. A time interval of $[-0.5 \mathrm{fs},-0.30 \mathrm{fs}]$ with a time step of $0.01 \mathrm{fs}$ is used. The CPU time is apparently proportional to the system size $N$.

We have calculated the absorption spectrum of a polyacetylene oligomer containing 10000 carbon atoms. The resulting absorption spectrum is plotted in Figure 1d. The optical gap is $2.08 \mathrm{eV}$. The inset shows the optical gap against $N$. The optical gap reduces drastically as $N$ increases. It saturates at $N \approx 200$.

\section{Carbon Nanotubes}

Our objective is to investigate the linear optical response of the zigzag CNTs by using the FMM-LDM method. The linear optical spectra of a series of open single-walled zigzag CNTs are evaluated. All the nearest-neighbor $\mathrm{C}-\mathrm{C}$ distances $a_{\mathrm{C}-\mathrm{C}}$ are 


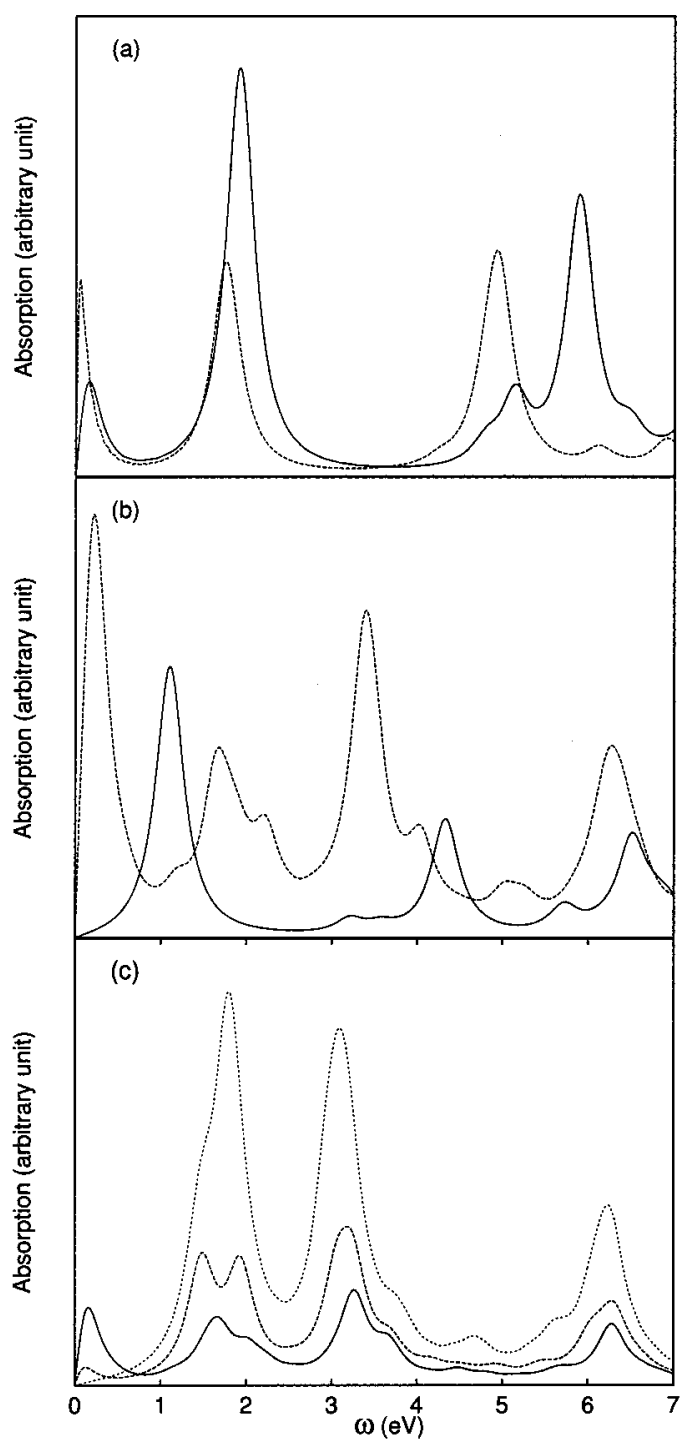

Figure 2. Absorption spectra of the zigzag $(8,0)$ CNTs with $\gamma=0.2$ eV. (a) $\mathrm{C}_{64} \mathrm{H}_{16}$ calculated using the PPP model and PM3 Hamiltonian. The solid line is for the PPP model, and the dashed line for the PM3 Hamiltonian. The same geometry is used in two calculations. (b) $\mathrm{C}_{N} \mathrm{H}_{16}$ calculated using the full TDHF method. The solid line is for $N=128$ and the dashed line for $N=256$. (c) Absorption spectrum of $\mathrm{C}_{320} \mathrm{H}_{16}$, $\mathrm{C}_{512} \mathrm{H}_{16}$, and $\mathrm{C}_{1024} \mathrm{H}_{16}$ calculated using the FMM-LDM method with critical length $l_{1}=l_{0}=36 \AA$. The dotted line is for $N=1024$, the dashed line for $N=512$, and the solid line for $N=320$.

chosen to be $1.421 \AA$, which are the same as those in graphite. A tube diameter $D_{\mathrm{m}}$ is $\sqrt{3 m a_{\mathrm{C}-\mathrm{C}}} / \pi$ in the zigzag $(m, 0)$ nanotubes which are formed by wrapping a graphite sheet along the zigzag direction $(m, 0)$ with $m$ hexagons around the circumference.

To test the validity of our PPP Hamiltonian for CNTs, we employ the LDM/PM3 (the LDM with the PM3 Hamiltonian ${ }^{53}$ ) method and calculate the absorption spectra of a small size CNT, $\mathrm{C}_{64} \mathrm{H}_{16}$. The results are compared with those of the LDM/PPP (the LDM with the PPP Hamiltonian) calculation in Figure 2a. They agree with each other quite well in both relative oscillator strengths and the peak locations in the low-frequency range $(\omega$ $<4 \mathrm{eV}$ ). The differences appear in the higher frequency range $\omega>4 \mathrm{eV}$ because of $\sigma$ electrons. Thus, the optical responses of CNTs in the low-frequency range may be described by the PPP Hamiltonian.

Figure 2 shows the absorption spectra for $\mathrm{C}_{64} \mathrm{H}_{16}, \mathrm{C}_{128} \mathrm{H}_{16}$, $\mathrm{C}_{256} \mathrm{H}_{16}, \mathrm{C}_{320} \mathrm{H}_{16}, \mathrm{C}_{512} \mathrm{H}_{16}$, and $\mathrm{C}_{1024} \mathrm{H}_{16}(8,0)$ CNTs. The first peak lies at $1.45 \mathrm{eV}$ for $\mathrm{C}_{1024} \mathrm{H}_{16}$. In the calculation, the dephasing parameter $\gamma$ is set to $0.2 \mathrm{eV}$. Two-, three-, and fourlevel FMM algorithms are employed for $N=320,512$, and 1024 , respectively ( $N$ is the number of carbon atoms). The absorption spectra are similar in the high-frequency region $(\omega$ $>1.5 \mathrm{eV}$ ) for $N=320,512$, and 1024 with slight red shifts of the absorption peaks as $N$ increases. The spectral shape looks quite different in the low-frequency region. For instance, the first peak near zero for $N=320$ and $N=512$ disappears when $N$ increases to 1024 . The optical gap $(1.45 \mathrm{eV})$ for the $(8,0)$ tubule is larger than those of the tight binding calculations $(1.22$ $\mathrm{eV}^{54}$ and $1.19 \mathrm{eV}^{55}$ ) and that of the ab initio pseudopotential local density functional calculation $\left(0.62 \mathrm{eV}^{55}\right)$. An absorption peak was also observed and located at about $4.6 \mathrm{eV}$ for $N=$ 1024. It is in good agreement with the similar absorption peaks for graphite, which is attributed to the saddle point of transitions between the $\pi$ bands at the critical points $Q$ of the twodimensional Brillouin zone. ${ }^{56-58} \mathrm{We}$ also find the two absorption peaks near $\omega=5.6$ and $\omega=6.2 \mathrm{eV}$ which are observed in the EELS experiment. ${ }^{59,60}$ These two peaks are attributed to the collective excitation of $\pi$ electrons in the CNTs.

To understand the nature of the electronic excitations, we examine the induced density matrices $\delta \rho^{(1)}$ corresponding to the excitations of $\mathrm{C}_{320} \mathrm{H}_{16}$ at the frequencies $0.16,1.66$, and 3.27 $\mathrm{eV}$. We find that the ground-state density matrix is almost diagonal. The electronic excitations, however, induce the electron-hole pairs between atoms that are far apart. The excitation at $\omega=0.16 \mathrm{eV}$ includes mainly the electron-hole pairs between two ends of the tubule, and the excitation at $\omega=$ $1.66 \mathrm{eV}$ contains mostly the electron-hole pairs in the middle of the tubule. It is conceivable that the excitation at $1.66 \mathrm{eV}$ will gain more oscillator strength and red shift to the lower energy region as the size increases. This is consistent with the spectral shapes of $N=512$ and 1024 .

To understand the spectral evolution as $N$ increases, we have calculated the linear optical response of the zigzag $(8,0)$ nanotubes with short lengths. The results are shown in Figure 2a,b. The absorption spectrum changes drastically with the increasing size. The overall spectral shape red shifts and the amplitude of the first peak reduces as $N$ increases. For instance, the first peaks for $N=64$ in Figure 2a disappear when $N=$ 128. The investigation of the corresponding density matrices reveals that the first peak for $N=64$ comes from the electronhole pairs in the two ends. Two ends play an important role in the optical responses of the short nanotubes. The influence of the two ends as well as the relative strength of the first peak decreases as the size increases.

It was predicted by the tight binding calculation that the infinitely long $(8,0)$ CNT is a semiconductor while the $(9,0)$ CNT is a conductor. ${ }^{40}$ We have calculated the absorption spectra of several zigzag $(9,0)$ CNTs. Figure 3 shows the calculated absorption spectra for $(9,0) \mathrm{C}_{108} \mathrm{H}_{18}$ and $\mathrm{C}_{216} \mathrm{H}_{18}$ by the full TDHF method and for $\mathrm{C}_{360} \mathrm{H}_{18}$ and $\mathrm{C}_{576} \mathrm{H}_{18}$ by the FMM-LDM method. Different dephasing parameters are used as indicated in Figure 3. The first peaks lie at $0.34,0.07,1.01$, and $0.72 \mathrm{eV}$ for $N=108,216,360$, and 576, respectively. Note the large red shifts in Figure 3b. In Figure 3a, as $N$ varies from 108 to 216 , the whole spectrum red shifts, and the oscillator strength of the first peak decreases drastically. This is similar to behavior in the $(8,0)$ CNTs. The collective excitation of $\pi$ electrons in CNTs $(9,0)$ is observed at $\omega \approx 5.0-7.0 \mathrm{eV}$.

Carbon nanotubes have cylindrical shapes. The anisotropy effects of the absorption spectrum are shown in Figure 4. Parts $\mathrm{a}$ and $\mathrm{b}$ of Figure 4 show, respectively, the absorption spectra 

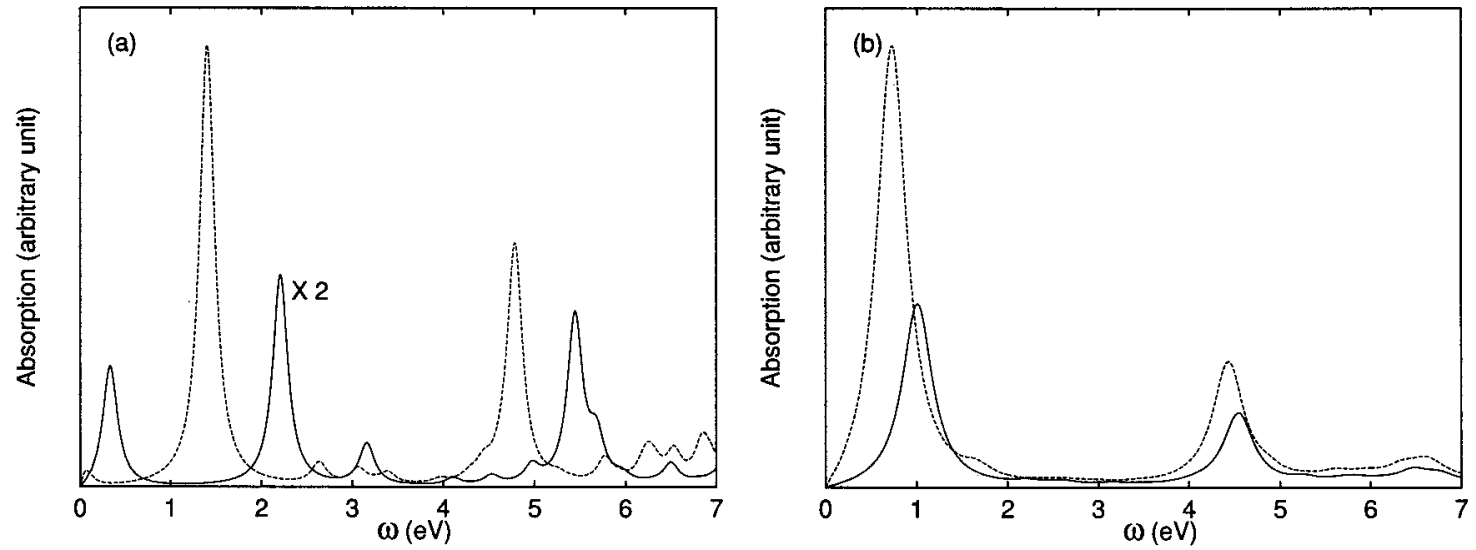

Figure 3. Absorption spectra of the zigzag $(9,0) \mathrm{CNTs}_{N} \mathrm{H}_{18}$. (a) The solid line is for $N=108$ and the dashed lien for $N=216$ with $\gamma=0.1$ $\mathrm{eV}$ calculated using the full TDHF method. The intensities of the absorption spectra are multiplied by 2 with $N=128$. (b) The solid line is for $N$ $=360$ and the dashed line for $N=576$ with $\gamma=0.2 \mathrm{eV}$.
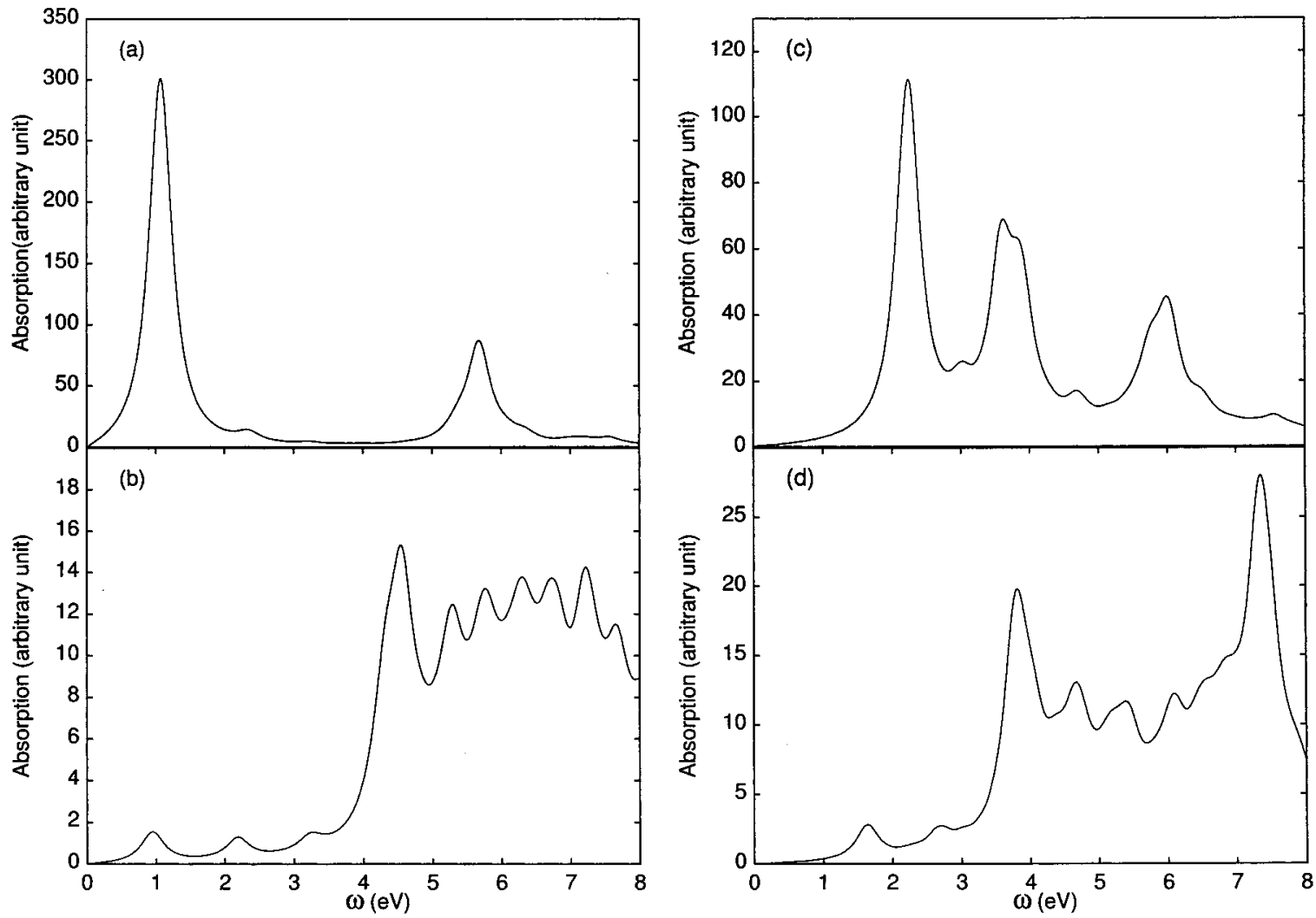

Figure 4. Optical absorption spectra of the zigzag $(6,0) \mathrm{CNTs}_{240} \mathrm{H}_{12}$ (a and b) and (7, 0) $\mathrm{CNTs}_{280} \mathrm{H}_{14}$ (c and d). In (a) and (c) the electronic field is parallel to the tube axis. In (b) and (d) the electronic field is perpendicular to the tube axis. $\gamma=0.2 \mathrm{eV}$. The FMM-LDM method is employed.

of a $(6,0) \mathrm{C}_{240} \mathrm{H}_{12}$ and a $(7,0) \mathrm{C}_{280} \mathrm{H}_{14}$, where the electronic field $\vec{E}$ is parallel to the tubule axis, while parts b and $\mathrm{d}$ of Figure 4 show the absorption spectra with $\vec{E}$ perpendicular to the axis. The spectral shapes are quite different for two different electric field directions. Figure $4 \mathrm{a}$ shows that the first peak at $1.07 \mathrm{eV}$ has a very strong oscillator strength while the first peak moves to $0.95 \mathrm{eV}$ with a much weaker peak when $\vec{E}$ is vertical to the axis (see Figure $4 \mathrm{~b}$ ). There are three peaks at $5-8 \mathrm{eV}$ with $\vec{E}$ along the axis. More peaks appear when the electronic field is vertical to the axis, and these peaks are similar to those of $\mathrm{C}_{70}{ }^{61,62}$ Parts $\mathrm{c}$ and $\mathrm{d}$ of Figure 4 also show different spectra. It is found that the absorption threshold is significantly higher (by about $0.12 \mathrm{eV}$ in the $(6,0)$ tubule and $0.60 \mathrm{eV}$ in the $(7,0)$ tubule) for the electronic field $\vec{E}$ along the tubule axis than for that vertical to the axis. The optical anisotropy of dispersed
CNTs induced by an electric field has been observed in the experiment, ${ }^{63}$ and may be used to determine the orientational ordering of the CNTs.

We compare the calculated optical absorption spectra of CNTs $(6,0) \mathrm{C}_{240} \mathrm{H}_{12},(7,0) \mathrm{C}_{280} \mathrm{H}_{14},(8,0) \mathrm{C}_{320} \mathrm{H}_{16}$, and $(9,0) \mathrm{C}_{360} \mathrm{H}_{18}$. These tubes are of the same length. $(7,0)$ and $(8,0)$ or $(6,0)$ and $(9,0)$ tubules have similar spectral shape. For $(7,0)$ and $(8,0)$ tubes, the larger the diameter, the smaller the gap. The peaks at about $\omega=5.0-6.5 \mathrm{eV}$ in all the four tubes are identified as the collective excitations of the $\pi$ band, which are consistent with EELS. ${ }^{59,60}$ These excitations are independent of the tubular chirality.

Carbon nanotubes are usually one-dimensional (1D) nanostructures since their lengths are far larger than their diameters. To illustrate that the FMM-LDM method is applicable to three- 

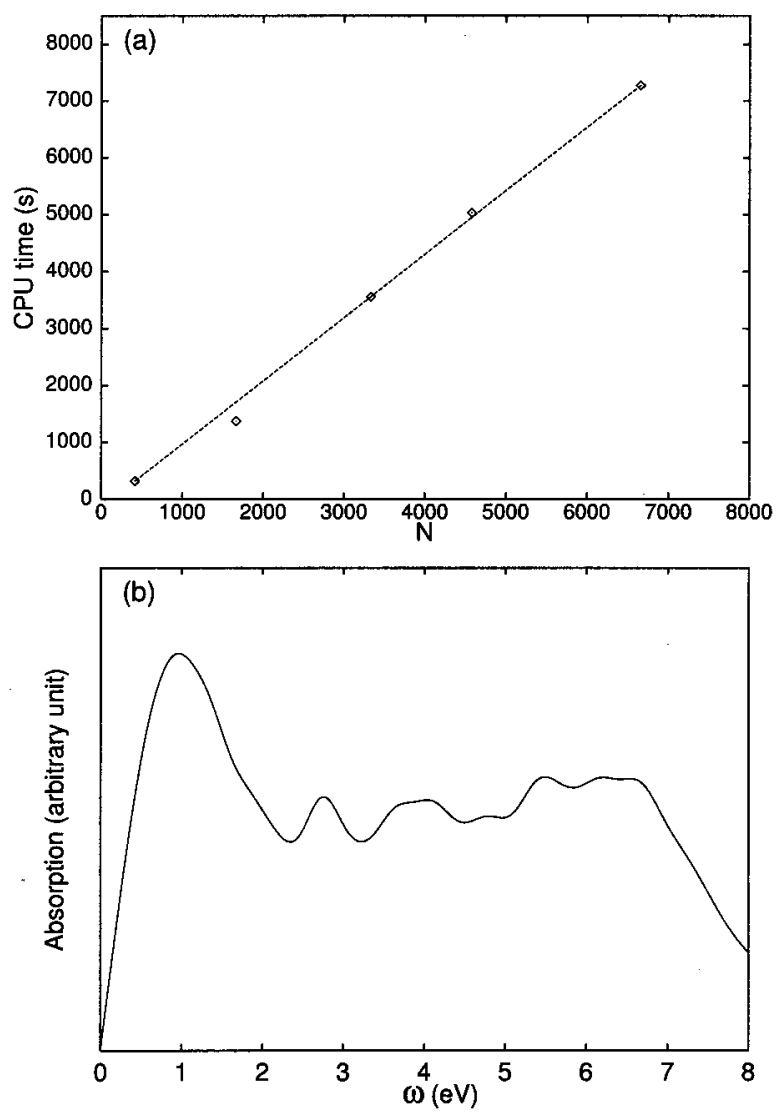

Figure 5. (a) CPU time for the excited-state calculation of threedimensional CNTs with a large radius. The time interval is $[-0.5 \mathrm{fs}$, $-0.3 \mathrm{fs}$ ] with the time step $0.01 \mathrm{fs}$. The critical length $l_{1}$ and $l_{0}$ are 15 $\AA$. Twenty-six atoms are included in the smallest box. (b) Absorption spectrum of the zigzag $(60,0)$ nanotube $\mathrm{C}_{1200} \mathrm{H}_{120} \cdot \gamma=0.4 \mathrm{eV}$.

dimensional (3D) systems, we consider the nanotubes with diameters comparable to their lengths. The diameters range from 20.370 to $81.478 \AA$, and the lengths from 15.63 to $66.78 \AA$. The number of carbon atoms corresponds to 416, 1664, 3328, 4576, and 6656. The CPU time for propagating eq 20 between a time interval of $[-0.5 \mathrm{fs},-0.3 \mathrm{fs}]$ is recorded. The time step is $0.01 \mathrm{fs}$. The critical lengths $l_{0}$ and $l_{1}$ are set to be $15 \AA$. The results are shown in Figure 5a. The CPU time scales linearly with the system size $N$. The maximum number of atoms in the smallest box is kept at 26.

The absorption spectrum of a CNT with 1200 carbon atoms is shown in Figure 5b. The critical lengths $l_{0}=28 \AA$ and $l_{1}=$ $43 \AA$ are used. The absorption spectrum is quite different from those of 1D CNTs. It should be similar to the optical properties of graphite ${ }^{57}$ if the tubule radius is large enough.

\section{Discussion and Conclusion}

We observe that for small zigzag CNTs $(N \lesssim 200)$ there is a small absorption peak at the very low energy region $(\lessgtr 0.3$ $\mathrm{eV}$ ) and the peak red shifts with decreasing oscillation strength as the size increases. These electronic excitations correspond to electron-hole pairs between two ends of the tubule, and appear to be caused by finite size effects. The critical behavior of the polyacetylene oligomer, for instance, the optical gap versus $N$, can be investigated, since the FMM-LDM may be applied to very large systems. The linear-scaling FMM-LDM method is quite general. It is applicable to $1 \mathrm{D}$ as well as 2D and 3D systems. In addition, it yields more accurate results than the cutoff-LDM method even for structured molecules such as polyacetylene oligomers. For an unstructured system it is expected to lead to better results than the cutoff-LDM method.

In the LDM/PPP calculation we consider only the $\pi$ electron in the CNTs. However, the curvature of the tubule leads to the hybridization of $\sigma$ and $\pi$ orbitals. The smaller the tubule, the more the hybridization of the orbitals. This hybridization may alter significantly the zero-order band structure of the systems. ${ }^{55}$ Therefore, it is desirable to include explicitly all valence orbitals.

To conclude, the FMM-LDM method has been developed and implemented to calculate the absorption spectra of very large polyacetylene oligomers and CNTs. It is demonstrated for the first time that the linear-scaling LDM method is applicable to calculation of the excited-state properties of two- or threedimensional systems. Interesting features of the absorption spectra of CNTs, for instance, low-frequency peaks and anisotropy, have been observed, and the corresponding electronhole excitations are identified. The influence of the finite length and chiral symmetry on the optical properties of CNTs has been investigated. We find the length and chiral symmetry of nanotubes have a strong influence on the low-energy optical excitations. In the high-frequency range $\omega=5-7 \mathrm{eV}$, the two peaks are observed, which correspond to the collective excitations of the $\pi$ electron and are independent of the tubular chirality.

Acknowledgment. Support from the Hong Kong Research Grant Council (RGC) and the Committee for Research and Conference Grants (CRCG) of the University of Hong Kong is gratefully acknowledged.

\section{Appendix}

We describe the FMM procedure that is employed to evaluate our effective Coulomb interaction in the calculation. The procedure is similar to that of ref 8 . The only difference arises from the special form of the effective Coulomb interaction that we adopt, the Ohno formula. ${ }^{47}$

The physical space is arranged in a hierarchy. The box containing the entire physical space is called the root or level 0 . Then each side of the root is bisected, and the space is divided into 8 smaller boxes. These smaller boxes are called the level 1 of hierarchy. The space may be further divided evenly until it is consisted of $8^{l}$ small boxes. The $8^{l}$ boxes form the level $l$ of hierarchy. The boxes at the same level of hierarchy that share a boundary point are said to be neighbors, and those that are not neighbors are said to be separated.

The Coulomb potential of all other charges to charge $i$ may be divided into two parts

$$
U\left(\vec{r}_{i}\right)=\sum_{k \in \mathrm{far}} U\left(\vec{r}_{i}-\vec{r}_{k}\right)+\sum_{j \in \operatorname{near}\left(a_{0}^{2}+\left|\vec{r}_{i}-\vec{r}_{j}\right|^{2}\right)^{1 / 2}} \frac{q_{j}}{a^{2}}
$$

where $q_{j}$ is the effective charge at $\vec{r}_{j}$. The first term is the farfield interaction, and the second is the near-field interaction. The near-field interaction is defined as the interaction between two charges inside a given box or neighboring boxes, and is treated exactly to ensure high accuracy. The far-field interaction is defined as the interaction between two charges in two separated boxes, and may be evaluated through the Taylor series expansion around the central position of the box containing charge $i$ as follows: 


$$
\begin{aligned}
V\left(\vec{r}_{i}^{\prime}\right) \equiv \sum_{k \in \mathrm{far}} U\left(\vec{r}_{i}-\vec{r}_{k}\right)=V_{0}+\sum_{\alpha} V_{\alpha} r_{i \alpha}{ }^{\prime}+ \\
\sum_{\alpha \beta} V_{\alpha \beta} r_{i \alpha}{ }^{\prime} r_{i \beta}{ }^{\prime}+\sum_{\alpha \beta \gamma} V_{\alpha \beta \gamma} r_{i \alpha}{ }^{\prime} r_{i \beta}{ }^{\prime} r_{i \gamma}{ }^{\prime}+\ldots
\end{aligned}
$$

where

$$
\begin{gathered}
V_{0}=\left.V\right|_{r_{i}^{\prime}=0} \\
V_{\alpha}=\left.\frac{\partial V}{\partial r_{i \alpha}{ }^{\prime}}\right|_{r_{i}^{\prime}=0} \\
V_{\alpha \beta}=\left.\frac{1}{2} \frac{\partial^{2} V}{\partial r_{i \alpha}{ }^{\prime} \partial r_{i \beta}{ }^{\prime}}\right|_{r_{i}^{\prime}=0} \\
V_{\alpha \beta \gamma}=\left.\frac{1}{6} \frac{\partial^{3} V}{\partial r_{i \alpha}{ }^{\prime} \partial r_{i \beta}{ }^{\prime} \partial r_{i \gamma}{ }^{\prime \prime}}\right|_{r_{i}^{\prime}=0}
\end{gathered}
$$

Here $\vec{r}_{i}^{\prime}=\vec{r}_{i}-\vec{R}_{m} . \vec{R}_{m}$ is the central position of the box $m$ on which charge $i$ is located. $\alpha, \beta$, and $\gamma$ are the indices representing $x, y$, and $z$. To calculate the Taylor coefficients, the Coulomb potential of all atoms in a particular box may be represented by the multipole moments of the box. Thus, the potential of charges in the far field to charge $i$ may be expressed as

$$
\begin{aligned}
& V\left(\vec{r}_{i}^{\prime}\right)=\sum_{n \in \text { far box }}\left\{\frac{Z^{n}}{\left(a_{0}^{2}+\left|\vec{r}_{i}^{\prime}-\vec{R}_{m n}\right|^{2}\right)^{1 / 2}}-\right. \\
& \frac{S^{n}}{\left(a_{0}^{2}+\left|\vec{r}_{i}^{\prime}-\vec{R}_{m n}\right|^{2}\right)^{5 / 2}}+\sum_{\alpha}\left[\frac{\mu_{\alpha}^{n}\left(\vec{r}_{i}^{\prime}-\vec{R}_{m n}\right)_{\alpha}}{\left(a_{0}{ }^{2}+\left|\vec{r}_{i}^{\prime}-\vec{R}_{m n}\right|^{2}\right)^{3 / 2}}-\right. \\
& \left.\frac{T_{\alpha}^{n}\left(\vec{r}_{i}^{\prime}-\vec{R}_{m n}\right)_{\alpha}}{\left(a_{0}^{2}+\left|\vec{r}_{i}^{\prime}-\vec{R}_{m n}\right|^{2}\right)^{7 / 2}}\right]+ \\
& \sum_{\alpha \beta} \frac{\left(\vec{r}_{i}^{\prime}-\vec{R}_{m n}\right)_{\alpha}\left(\vec{r}_{i}^{\prime}-\vec{R}_{m n}\right)_{\beta} Q_{\alpha \beta}^{n}}{\left(a_{0}^{2}+\left|\vec{r}_{i}^{\prime}-\vec{R}_{m n}\right|^{2}\right)^{5 / 2}}+ \\
& \left.\sum_{\alpha \beta \gamma} \frac{\left(\vec{r}_{i}^{\prime}-\vec{R}_{m n}\right)_{\alpha}\left(\vec{r}_{i}^{\prime}-\vec{R}_{m n}\right)_{\beta}\left(\vec{r}_{i}^{\prime}-\vec{R}_{m n}\right)_{\gamma} Q_{\alpha \beta \gamma}^{n}}{\left(a_{0}^{2}+\left|\vec{r}_{i}^{\prime}-\vec{R}_{m n}\right|^{2}\right)^{7 / 2}}+\ldots\right\}
\end{aligned}
$$

Here, $\vec{R}_{m n}=\vec{R}_{n}-\vec{R}_{m}$ is the displacement vector from the center of box $m$ to the center of box $n$. We calculate the Taylor series expansion coefficients of the field at the center of box $m$ due to the multipole moments $Z^{n}, S^{n}, \mu^{n}, T^{n}, Q^{n}$, and $O^{n}$ of the boxes $n$.

The boxes in the far field may be further divided into two groups: One contains the boxes that belong to the neighbors of $m$ 's parent except $m$ 's neighbors (denoted as pnc). Another includes the area outside the 27 neighbor boxes of $m$ 's parent (denoted as pfc). Thus

$$
V\left(\vec{r}_{i}^{\prime}\right)=V^{\mathrm{pnc}}\left(\vec{r}_{i}^{\prime}\right)+V^{\mathrm{pfc}}\left(\vec{r}_{i}^{\prime}\right)
$$

where $V^{\mathrm{pnc}}\left(\vec{r}_{i}^{\prime}\right)$ and $V^{\mathrm{pfc}}\left(\vec{r}_{i}^{\prime}\right)$ are of the form in eq $30 . V^{\mathrm{pnc}}\left(\vec{r}_{i}^{\prime}\right)$ is evaluated at the individual box at the same level as $m$. The Taylor series expansion coefficients $[V]$ in eq 25 according to the center of box $m$ coming from the contribution of the far field is just the summation of the two expansion coefficients $\left[V^{p n c}\right]$ and $\left[V^{p f c}\right]$, for example

$$
\begin{aligned}
& V_{0}=\sum_{n \in \mathrm{pnc}}\left[\frac{Z^{n}}{\left(a_{0}^{2}+R^{2}\right)^{1 / 2}}-\frac{S^{n}}{\left(a_{0}^{2}+R^{2}\right)^{5 / 2}}+\frac{\vec{\mu}^{n} \cdot \vec{R}}{\left(a_{0}^{2}+R^{2}\right)^{3 / 2}}-\right. \\
& \left.\frac{\vec{T}^{n} \cdot \vec{R}}{\left(a_{0}^{2}+R^{2}\right)^{7 / 2}}+\sum_{\mu \nu} \frac{Q_{\mu \nu}^{n} R_{\mu} R_{\nu}}{\left(a_{0}^{2}+R^{2}\right)^{5 / 2}}+\sum_{\mu \nu \lambda} \frac{O_{\mu \nu \lambda}^{n} R_{\mu} R_{v} R_{\lambda}}{\left(a_{0}^{2}+R^{2}\right)^{7 / 2}}+\ldots\right]+ \\
& V^{\mathrm{P}}+\sum_{\alpha} V_{\alpha}^{\mathrm{P}} r_{\mathrm{pc} \alpha}+\sum_{\alpha \beta} V_{\alpha \beta}^{\mathrm{P}} r_{\mathrm{pc} \alpha} r_{\mathrm{pc} \beta}+\sum_{\alpha \beta \gamma} V_{\alpha \beta \gamma}^{\mathrm{P}} r_{\mathrm{pc} \alpha} r_{\mathrm{pc} \beta} r_{\mathrm{pc} \gamma} \\
& V_{\alpha}=\sum_{n \in \mathrm{pnc}}\left[-\frac{Z^{n} R_{\alpha}}{\left(a_{0}^{2}+R^{2}\right)^{3 / 2}}+5 \frac{S^{n} R_{\alpha}}{\left(a_{0}^{2}+R^{2}\right)^{7 / 2}}+\right. \\
& \frac{\mu_{\alpha}^{n}}{\left(a_{0}^{2}+R^{2}\right)^{3 / 2}}-3 \frac{\left(\vec{\mu}^{n} \cdot \vec{R}\right) R_{\alpha}}{\left(a_{0}^{2}+R^{2}\right)^{5 / 2}}-\frac{T_{\alpha}^{n}}{\left(a_{0}^{2}+R^{2}\right)^{7 / 2}}+ \\
& 7 \frac{\left(\vec{T}^{n} \cdot \vec{R}\right) R_{\alpha}}{\left(a_{0}^{2}+R^{2}\right)^{9 / 2}}+\frac{2 \sum_{\nu} Q_{\alpha \nu}^{n} R_{v}}{\left(a_{0}^{2}+R^{2}\right)^{5 / 2}}-5 \frac{\sum_{\mu \nu} Q_{\mu \nu}^{n} R_{\mu} R_{v} R_{\alpha}}{\left(a_{0}^{2}+R^{2}\right)^{7 / 2}}+ \\
& \left.3 \frac{\sum_{\mu \nu} O_{\alpha \mu \nu}^{n} R_{\mu} R_{v}}{\left(a_{0}^{2}+R^{2}\right)^{7 / 2}}-7 \frac{\sum_{\mu \nu \lambda} O_{\mu \nu \lambda}^{n} R_{\mu} R_{v} R_{\lambda} R_{\alpha}}{\left(a_{0}^{2}+R^{2}\right)^{9 / 2}}+\ldots\right]+V_{\alpha}^{\mathrm{P}}+ \\
& 2 \sum_{\beta} V_{\alpha \beta}^{\mathrm{P}} r_{p c \beta}+3 \sum_{\beta \gamma} V_{\alpha \beta \gamma}^{\mathrm{P}} r_{p c \beta} r_{p c \gamma}
\end{aligned}
$$

Here, $\vec{R}=\vec{R}_{n m}$ and $V^{\mathrm{P}}, V_{\alpha}^{\mathrm{P}}, V_{\alpha \beta}^{\mathrm{P}}$, and $V_{\alpha \beta \gamma}^{\mathrm{P}}$ are the Taylor series coefficients of cell $m$ 's parent cell. The Taylor series coefficients coming from the pfc cells' contribution have been represented by the coefficients of cell $m$ 's parent cell. We just translate those coefficients with respect to the center of its parent box to the center of the box which the charge $i$ occupies. $\vec{r}_{\mathrm{pc}}$ is the displacement vector from the center of its parent box to the box which the charge $i$ occupies. Those Taylor series coefficients may be calculated level by level, from the root to the leaves.

The multipole moments, i.e., charges, dipoles, quadrupoles, octapoles, etc., in box A, may be evaluated as follows:

$$
\begin{aligned}
& Z=\sum_{k \in \mathrm{A}} q_{k} \\
& S=a_{0}^{2} \sum_{k \in \mathrm{A}}(1 / 2) q_{k} r_{k}^{2} \\
& \mu_{\alpha}=\sum_{k \in \mathrm{A}} q_{k} r_{k \alpha}{ }^{\prime} \\
& T_{\alpha}=a_{0}{ }^{2} \sum_{k \in \mathrm{A}}(3 / 2) q_{k} r_{k}{ }^{\prime 2} r_{k \alpha}{ }^{\prime} \\
& Q_{\alpha \beta}=\sum_{k \in \mathrm{A}}(1 / 2) q_{k}\left[3 r_{k \alpha} r_{k \beta}{ }^{\prime}-r_{k}^{\prime 2} \delta_{\alpha \beta}\right] \\
& O_{\alpha \beta \gamma}=\sum_{k \mathrm{EA}}(1 / 2) q_{k}\left[5 r_{k \alpha}{ }^{\prime} r_{k \beta}{ }^{\prime} r_{k \gamma}{ }^{\prime}-\right. \\
& \left.\left(\delta_{\alpha \beta} r_{k \gamma}{ }^{\prime}+\delta_{\alpha \gamma} r_{k \beta}{ }^{\prime}+\delta_{\gamma \beta} r_{k \alpha}{ }^{\prime}\right) r_{k}{ }^{2}\right]
\end{aligned}
$$

here, $\vec{r}_{k}^{\prime}$ is the displacement vector from the center A to the charge $k$. The contributions of these multipole moments of different boxes at various levels are summed up and further expanded in $\vec{r}$ to obtain the values of Taylor coefficients $V_{0}$, $V_{\alpha}, V_{\alpha \beta}, V_{\alpha \beta \gamma}$, and others. Once the multipole moments of the highest level are known, the multipole moments of other levels may be calculated readily by utilizing the relationship between the multipole moments of level $l$ and level $l-1$. 


$$
\begin{gathered}
Z^{l-1}=\sum_{k \in \mathrm{C}_{l-1}} q_{k}=\sum_{l \in \mathrm{C}_{l-1}} \sum_{k \in \mathrm{C}_{l}} q_{k}=\sum_{l \in \mathrm{C}_{l-1}} Z^{l} \\
S^{l-1}=(1 / 2) a_{0}^{2} \sum_{l \in \mathrm{C}_{l-1}} Z^{l} r_{l}^{2}+\sum_{l \in \mathrm{C}_{l-1}} S^{l}+a_{0}^{2} \sum_{l \in \mathrm{C}_{l-1}}\left(\vec{\mu}^{l} \cdot \vec{r}_{l}\right) \\
\mu_{\alpha}^{l-1}=\sum_{l \in \mathrm{C}_{l-1}} Z^{l} r_{l \alpha}+\sum_{l \in \mathrm{C}_{l-1}} \mu_{\alpha}^{l} \\
T_{\alpha}^{l-1}=\sum_{l \in \mathrm{C}_{l-1}} T_{\alpha}^{l}+4 \sum_{l \in \mathrm{C}_{l-1}} S_{l \alpha}^{l} r_{l \alpha}+ \\
\sum_{l \in \mathrm{C}_{l-1}}(3 / 2) a_{0}^{2} r_{\mathrm{pc}}^{l}\left(\mu_{\alpha}^{l}+Z^{l} r_{l \alpha}\right)+\sum_{l \in \mathrm{C}_{l-1}} 2 a_{0}^{2} \sum_{\lambda} Q_{\lambda \alpha}^{l} r_{l \lambda} \\
Q_{\alpha \beta}^{l-1}=(1 / 2) \sum_{l \in C_{l-1}} Z^{l}\left[3 r_{l \alpha} r_{l \beta}-r_{l}^{2} \delta_{\alpha \beta}\right]+\sum_{l \in C_{l-1}} Q_{\alpha \beta}^{l}+ \\
(1 / 2) \sum_{l \in C_{l-1}}\left[3\left(\mu_{\alpha}^{l} r_{l \beta}+\mu_{\beta}^{l} r_{l \alpha}\right)-2\left(\vec{\mu}^{l} \cdot \vec{r}_{l}\right) \delta_{\alpha \beta}\right] \\
O_{\alpha \beta \gamma}^{l-1}=\sum_{l \in \mathrm{C}_{l-1}}(1 / 2) Z^{l}\left[5 r_{l \alpha} r_{l \beta} r_{l \gamma}-\left(\delta_{\alpha \beta} r_{l \gamma}+\delta_{\alpha \gamma} r_{l \beta}+\right.\right. \\
\left.\left.\delta_{\gamma \beta} r_{l \alpha}\right) r_{l}^{2}\right]+\sum_{l \in \mathrm{C}_{l-1}}(5 / 2)\left(\mu_{\alpha}^{l} r_{l \beta} r_{l \gamma}+\mu_{\beta}^{l} r_{l \alpha} r_{l \gamma}+\mu_{\gamma}^{l} r_{l \beta} r_{l \alpha}\right)+ \\
\sum_{l \in \mathrm{C}_{l-1}}(5 / 3)\left(Q_{\alpha \beta}^{l} r_{l \gamma}+Q_{\alpha \gamma}^{l} r_{l \beta}+Q_{\gamma \beta}^{l} r_{l \alpha}\right)- \\
\sum_{l \in \mathrm{C}_{l-1}}\left(\vec{\mu}^{l} \cdot \vec{r}_{l}\right)\left(\delta_{\alpha \beta} r_{l \gamma}+\delta_{\alpha \gamma} r_{l \beta}+\delta_{\gamma \beta} r_{l \alpha}\right)- \\
\sum_{l \in \mathrm{C}_{l-1}}(1 / 2) r_{l}^{2}\left(\delta_{\alpha \beta} u_{\gamma}^{l}+\delta_{\alpha \gamma} \mu_{\beta}^{l}+\delta_{\gamma \beta} \mu_{\alpha}^{l}\right)- \\
\sum_{l \in \mathrm{C}_{l-1}}(2 / 3)\left(\delta_{\alpha \beta} \sum_{\lambda} Q_{\gamma \lambda}^{l} r_{l \lambda}+\delta_{\gamma \beta} \sum_{\lambda} Q_{\alpha \lambda}^{l} r_{l \lambda}+\right. \\
\left.\delta_{\alpha \gamma} \sum_{\beta \lambda} Q_{l \lambda}^{l}\right)+\sum_{l \in \mathrm{C}_{l-1}} O_{\alpha \beta \gamma}^{l}(43)
\end{gathered}
$$

where $\vec{r}_{l}$ is the displacement vector from the center of a box at level $l$ to the center of box $C_{l-1}$ at level $l-1$.

\section{References and Notes}

(1) Yokojima, S.; Chen, G. H. Chem. Phys. Lett. 1998, 292, 379.

(2) Yokojima, S.; Chen, G. H. Phys. Rev. B 1999, 59, 7259.

(3) Yokojima, S.; Chen, G. H. Chem. Phys. Lett. 1999, 300, 540.

(4) Yokojima, S.; Zhou, D. H.; Chen, G. H. Chem. Phys. Lett. 1999, 302,495 .

(5) Liang, W. Z.; Yokojima, S.; Chen, G. H. J. Chem. Phys. 1999, 110,1844 .

(6) Ring, P.; Schuck, P. The Nuclear Many-Body Problem; Springer: New York, 1980.

(7) Ding, H.-Q.; Karasawa, N.; Goddard, W. A., III. Chem. Phys. Lett. 1992, 196, 6 .

(8) Ding, H.-Q.; Karasawa, N.; Goddard, W. A., III. J. Chem. Phys. 1992, 97, 4309.

(9) Appel, A. W. SIAM J. Sci. Stat. Comput. 1985, 6, 85.

(10) Barnes, J. E.; Hut, P. Nature 1986, 324, 446.

(11) Greengard L.; Rokhlin, V. J. Comput. Phys. 1987, 73, 325.

(12) Greengard, L. The Rapid Evaluation of Potential, Fields in Particle Systems; MIT Press: Cambridge, MA, 1988.

(13) White, C. A.; Johnson, B. G.; Gill P. M. W.; Head-Gordon, M. Chem. Phys. Lett. 1996, 253, 268.

(14) Strain, M. C.; Scuseria, G. E.; Frisch, M. J. Science 1996, 271, 51.

(15) Marder, S. R.; Perry, J. W.; Bourhill, G.; Gorman, C. B.; Tiemann,

B. G.; Mansour, K. Science 1993, 261, 186.

(16) Samuel, I. D. W.; Ledoux, I.; Dhenaut, C.; Zyss, J.; Fox, H. H.; Schrock, R. R.; Silbey, R. J. Science 1994, 265, 1070.

(17) Garito, A.; Shi, R. F.; Wu, M. Phys. Today 1994, May, 51.

(18) Etemad, S.; Soos, Z. G. In Spectroscopy of Advanced Materials;

Clark, R. J. H., Hester, R. E., Eds.; Wiley: New York, 1991; p 87.

(19) (a) Takahashi, A.; Mukamel, S. J. Chem. Phys. 1994, 100, 2366.

(b) Chen, G. H.; Mukamel, S. J. Am. Chem. Soc. 1995, 117, 4945.

(20) Fukutome, H. J. Mol. Struct.: THEOCHEM 1989, 188, 337.

(21) Iijima, S. Nature 1991, 354, 56.
(22) Treacy, M. M. J; Ebbesen, T. W.; Gibson, J. M. Nature 1996, 381, 678.

(23) Dresselhaus, M. S.; Dresseihaus, G.; Eklund, P. C. Science of Fullerenes and Carbon Nanotubes; Academic Press: San Diego, 1996.

(24) Ge, M.; Sattler, K. Appl. Phys. Lett. 1994, 65, 2284.

(25) Wildöer, J. W. G.; Venema, L. C.; Rinzler, A. G.; Smalley, R. E.; Dekker, C. Nature 1998, 391, 59.

(26) Odom, T. W.; Huang, J.-L.; Kim P.; Lieber, C. M. Nature 1998, 391,62 .

(27) Wong, E. W.; Sheehan P. E.; Lieber, C. M. Science 1997, 277, 1971.

(28) Olk, C. H.; Heremans, J. P. J. Mater. Res. 1994, 9, 259.

(29) Benedict, L. X.; Crespi, V. H.; Louie, S. G.; Cohen, M. L. Phys. Rev. B 1995, 52, 14935.

(30) Lin, M. F.; Shung, K. W.-K. Phys. Rev. B 1995, 52, 8423.

(31) Lin, M.-F. J. Phys. Soc. Jpn. 1998, 67, 1094.

(32) Tamura, R.; Tsukada, M. Phys. Rev. B 1998, 58, 8120.

(33) Langer, L.; Bayot, V.; Grivei, E.; Issi, J.-P.; Heremans, J. P.; Olk, C. H.; Stockman, L.; Van Haesendonck, C.; Bruynseraede, Y. Phys. Rev. Lett. 1996, 76, 479.

(34) Iijima S.; Ichihashi, T. Nature 1993, 363, 603.

(35) Holden, J. M.; Zhou, P.; Bi, X.-X.; Eklund, P. C.; Bandow, S.; Jishi, R. A.; Das Chowdhury, K.; Dresselhaus, G.; Dresselhaus, M. S. Chem. Phys. Lett. 1994, 220, 186.

(36) Hiura, H.; Ebbesen T. W.; Tanigaki, K.; Takahashi, H. Chem. Phys. Lett. 1993, 202, 509.

(37) Rao, A. M.; Richter, E.; Bandow S.; Chase B.; Eklund, P. C.; Williams, K. A.; Fang, S.; Subbaswamy, K. R.; Menon, M.; Thess, A.; Smalley, R. E.; Dresselhaus, G.; Dresselhaus, M. S. Science 1997, 275, 187.

(38) Rinzler, A. G.; Hafner, J. H.; Nikolaev, P.; Lou, L.; Kim, S. G.; Tomrnek, D.; Nordlander, P.; Colbert, D. T.; Smalley, R. E. Science 1995 269,1550 .

(39) de Heer, W. A.; Chatelain, A.; Ugarte, D. Science 1995, 270, 1179.

(40) (a) Saito, R.; Fujita, M.; Dresselhaus, G.; Dresselhaus, M. S. Appl. Phys: Lett. 1992, 60, 2204. (b) Saito, R.; Fujita, M.; Dresselhaus, G.; Dresselhaus, M. S. Phys. Rev. B 1992, 46, 1804.

(41) Dravid, V. P.; Lin, X.; Wang, Y.; Wang, X. K.; Yee, A.; Ketterson, J. B.; Chang, R. P. H. Science 1993, 259, 1601.

(42) Ajayan, P. M.; Iijima, S.; Ichihashi, T. Phys. Rev. B 1993, 47, 6859.

(43) Liu, X.; Si, J.; Chang, B.; Xu, G.; Yang, Q.; Pan, Z.; Xie, S.; Ye, P.; Fan, J.; Wan, M. Appl. Phys. Lett. 1999, 74, 164.

(44) Wan, X.; Dong, J. Phys. Rev. B 1998, 58, 6756.

(45) Ma, J.; Yuan, R.-K. Phys. Rev. B 1998, 57, 9343.

(46) Hasanein, A. A.; Evans, M. W. Computational Methods in Quantum Chemistry, Quantum Chemistry Vol. 2; World Scientific: Singapore, 1996.

(47) Ohno, K. Theor. Chim. Acta 1964, 2, 219.

(48) Balents, L.; Fisher, M. P. A. Phys. Rev. B 1997, 55, 11973.

(49) McWeeny, R. Rev. Mod. Phys. 1960, 32, 335.

(50) Li, X.-P.; Nunes, R. W.; Vanderbilt, D. Phys. Rev. B 1993, 47, 10891.

(51) Greengard, L. Science 1994, 265, 909.

(52) Press, W. H.; Flannery, B. P.; Teukolsky, S. A.; Vetterling, W. T. Numerical Recipes in C; Cambridge University Press: New York, 1988.

(53) Stewart, J. J. P. J. Comput. Chem. 1989, 10, 209.

(54) Hamada, N.; Sawada, S.; Oshiyama, A. Phys. Rev. Lett. 1992, 68, 1579.

(55) Blase, X.; Benedict, L. X.; Shirley, E. L.; Louie, S. G. Phys. Rev. Lett. 1994, 72, 1878.

(56) Taft, E. A.; Phiipp, H. R. Phys. Rev. 1965, 138, A197.

(57) Willis, R. F.; Feuerbacher, B.; Fitton, B. Phys. Rev. B 1971, 4, 2441.

(58) Johnson, L. G.; Dresselhaus, G. Phys. Rev. B 1973, 7, 2275.

(59) Lin, M. F.; Chuu, D. S. Phys. Rev. B 1998, 57, 10183.

(60) Pichler, T.; Kunpfer, M.; Golden, M. S.; Fink, J.; Rinzler, A.; Smalley, R. E. Phys. Rev. Lett. 1998, 80, 4729.

(61) Harigaya, K.; Abe, S. Phys. Rev. B 1994, 49, 16746.

(62) Shumway, J.; Satpathy, S. Chem. Phys. Lett. 1993, 211, 595.

(63) Bubke, K.; Gnewuch, H.; Hempstead, M.; Hammer, J.; Green, M. L. H. Appl. Phys. Lett. 1997, 71, 1906. 